\title{
MALE ANTIFERTILITY COMPOUNDS: STRUCTURE AND ACTIVITY RELATIONSHIPS OF U-5897, U-15,646 AND RELATED SUBSTANCES
}

\author{
R. J. ERICSSON AND G. A. YOUNGDALE
}

Fertility Research, The Upjohn Company, Kalamazoo, Michigan 49001

\author{
(Received 7th Fanuary 1969)
}

\begin{abstract}
Summary. U-5897 (3-chloro-1,2-propanediol), U-15,646 (1,1'-(pentamethylenedioxy) bis [3-chloro-2-propanol]), and some related compounds were found to have antifertility activity in the male rat. Oral administration for 8 days produced temporary sterility of a functional type in the male. Structural characteristics for activity were studied.
\end{abstract}

\section{INTRODUGTION}

Male antifertility research has, in the past, been dominated by scientists seeking ways to inhibit spermatogenesis. Other approaches seemed less attractive due to absence of positive information. The present approach to the problem was via the spermatozoa in the excurrent ducts. Advantages for induced sterility of post-testicular spermatozoa are: (i) almost immediate effectiveness; (ii) readily reversible; (iii) reduced chances of mutagenic damage; (iv) avoid psychological or endocrine impairment of libido; and (v) enhance knowledge of epididymal sperm maturation and fertility.

The overall objective is to find a drug(s) which when taken by the human male gives safe and effective contraception. Of immediate concern was the lack of any compound which approached this objective, even for laboratory experimentation. Empirical screening of numerous compounds resulted in the discovery that some chlorohydrins have antifertility properties in the male. This report is concerned with the relationship between chemical structure and antifertility.

\section{COMPOUNDS AND METHODS}

Compounds for test were purchased from Aldrich Ghemical Co. and Distillation Products Industry, or obtained from General Tire and Rubber Co. (U-15,646), or prepared by literature procedures. All compounds were administered, at varying doses, in $0.5 \mathrm{ml}$ of vehicle $122\left(0.25 \%\right.$ methylcellulose in sterile $\left.\mathrm{H}_{2} \mathrm{O}\right)$. The test animal was a $300 \pm 25 \mathrm{~g}$ Spartan or Upjohn male rat. Three rats per test received the compound daily for 8 days by oral intubation. Males selected to receive compounds were those which successfully mated the previous night with PMSG-primed, immature females (Uberoi \& Meyer, 1966). Oestrous females were caged overnight (Day 8) with test males and then checked for evidence of mating (spermatozoa in vagina and/or vaginal plugs). Males which 
did not mate were given another opportunity the following night (Ericsson \& Baker, 1966). Antifertility activity was assessed in terms of implantation sites found in mated females on Day 9 or 10 of gestation.

\section{RESULTS AND DISGUSSION}

Tables 1 and 2 give the antifertility effectiveness, at $30 \mathrm{mg} /$ rat dose, of U-5897 (3-chloro-1,2-propanediol), U-15,646 (1,1'-(pentamethylenedioxy) bis [3chloro-2-propanol]) and related compounds. The relative antifertility potency

TABLE 1

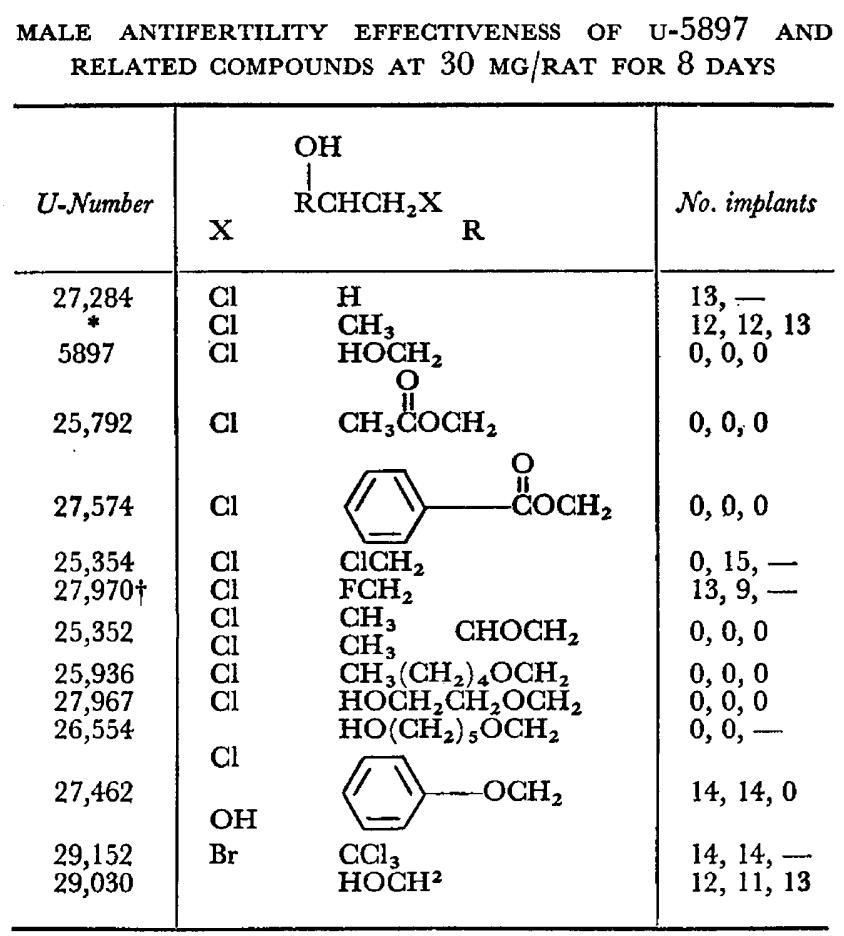

$(\rightarrow)$; did not mate.

${ }^{*} \mathrm{~A}$ mixture consisting of $85 \%$ 1-chloro-2-propanol and $12 \%$ 2-chloro-1-propanol.

$+5 \mathrm{mg} / \mathrm{rat}$ dose due to low $\mathrm{LD}_{50}$.

of these U-numbered compounds is given in Table 3. It should not be inferred from this table, however, that some compounds not listed are incapable of activity if tested at a high enough dose. Biological properties of U-5897 and U-15,646 in reproductive processes are given in separate papers (see Ericsson \& Baker, 1970; Ericsson, 1970; Kirton, Ericsson, Ray \& Forbes, 1970).

Examination of the tables reveals definite structural characteristics for activity. Active compounds (as defined by our test conditions) are chlorohydrins, i.e. compounds incorporating a carbon bearing a chlorine adjacent to a carbon bearing a hydroxyl group. An added characteristic is that the hydroxyl-bearing carbon is adjacent to another carbon bearing an oxygen either as a hydroxyl or 
an ether group; the hydroxyls can be free or esterified. The most active compound found was the simplest one possessing these features, namely, 3-chloro1,2-propanediol (U-5897). Incorporating two chlorohydrin groups in one compound, for example, 1,1'-(pentamethylenedioxy) bis [3-chloro-2-propanol] (U-15,646), resulted in active but less potent compounds than U-5897 (U-27,421, U-26,126, U-27,045, U-27,151). Compounds in which the hydroxyl group was esterified retained activity but were less potent than the unesterified compounds (U-25,792, U-27,574, U-26,853). Supporting these correlations are the inactivity of ethylene chlorohydrin (U-27,284), 1-chloro-2-propanol $(85 \%$ pure), 1-chloro-3-fluoro-2-propanol (U-27,970) and 1,3-dichloro-2-propanol (U-25,354) which are chlorohydrins lacking additional oxygen-bearing carbon, besides the inactivity of compounds in which the chlorine is replaced by fluorine, bromine, iodine, or a trichloro group (U-29,030, U-27,214, U-25,888, U-27,971, U-29,152). However, compounds may possess all the above struc-

TABle 2

MALE ANTIFERTILITY EFFECTIVENESS OF U-15,646 AND RELATED COMPOUNDS AT $30 \mathrm{MG} / \mathrm{RAT}$ FOR 8 DAYS

\begin{tabular}{|c|c|c|c|c|}
\hline$U$-Number & \multicolumn{3}{|c|}{$\stackrel{\mathrm{OR}}{\stackrel{\mathrm{OR}}{!} \mathrm{xCH}_{2} \mathrm{CHCH}_{2} \mathrm{OR}_{1} \mathrm{OCH}_{2} \mathrm{CHCH}_{2} \mathrm{X}}$} & No. implants \\
\hline & & & & \\
\hline $\begin{array}{l}27,421 \\
26,126\end{array}$ & $\mathrm{Gl}$ & $\mathrm{H}$ & $\left(\mathrm{CH}_{2}\right)_{2}$ & $0,0,-$ \\
\hline $\begin{array}{l}27,126 \\
27,824\end{array}$ & $\mathrm{Cl}$ & $\stackrel{\mathrm{H}}{\mathbf{H}}$ & $\begin{array}{l}\left(\mathrm{CH}_{2}\right)_{3} \\
\left(\mathrm{GH}_{2}\right)_{4}\end{array}$ & $17,13,12$ \\
\hline 15,646 & $\mathrm{Cl}$ & $\mathbf{H}$ & $\left(\mathrm{CH}_{2}\right)_{5}$ & $0,0,0$ \\
\hline 26,715 & $\mathrm{Cl}$ & $\overrightarrow{\mathrm{H}}$ & $\left(\mathrm{CH}_{2}\right)_{6}$ & $12,7,6$ \\
\hline 27,045 & $\mathrm{Cl}$ & $\mathbf{H}$ & $\left(\mathrm{CH}_{2}\right)_{10}$ & $0,0,0$ \\
\hline 27,214 & $\mathrm{Br}$ & $\mathbf{H}$ & $\left(\mathrm{CH}_{2}\right)_{5}$ & $14,14,-$ \\
\hline 27,971 & & & $\begin{array}{l}\left(\mathrm{CH}_{2}\right)_{5} \\
\left(\mathrm{CH}_{2}\right)_{5}\end{array}$ & $12,12,14$ \\
\hline 26,853 & $\mathrm{Cl}$ & $\mathrm{CH}_{3}{ }^{\prime \prime} \mathrm{C}$ & $\begin{array}{l}\left(\mathrm{CH}_{2}\right)_{5} \\
\mathrm{CH}_{3}\end{array}$ & $10,1,0$ \\
\hline 27,874 & $\mathrm{Cl}$ & $\mathbf{H}$ & $\mathrm{CHCH}_{2}$ & $1,2,4$ \\
\hline 27,963 & $\mathrm{Cl}$ & $\mathbf{H}$ & $\mathrm{CH}_{2} \mathrm{CH}_{2} \mathrm{CCH}_{2} \mathrm{CH}_{2}$ & $12,12,15$ \\
\hline $\begin{array}{l}27,151 \\
27,456\end{array}$ & $\begin{array}{l}\mathrm{Cl} \\
\mathrm{Cl}\end{array}$ & $\underset{\mathbf{H}}{\mathbf{H}}$ & $\begin{array}{l}\mathrm{CH}_{2} \mathrm{C}=\mathrm{CCH}_{2} \\
\mathrm{CH}_{2} \mathrm{CH}_{2} \mathrm{OCH}_{2} \mathrm{CH}_{2}\end{array}$ & $\begin{array}{l}0,0,-\overline{13} \\
9,8,\end{array}$ \\
\hline 27,873 & $\mathrm{Cl}$ & $\mathrm{H}$ & $-\mathrm{CH}_{2}$ & $1,9,4$ \\
\hline 26,123 & $\mathrm{Cl}$ & $\mathbf{H}$ & $-\mathrm{CH}_{2}$ & $12,14,-$ \\
\hline 26,527 & $\mathrm{Cl}$ & $\mathbf{H}$ & & $13,14,0$ \\
\hline
\end{tabular}


tural characteristics and be inactive (U-27,462, U-27,456, U-27,873, U-26,527, $\mathrm{U}-26,123)$.

TABLE 3

POTENCY OF U-NUMBERED GOM-

POUNDS IN MALE ANTIFERTILITY

TEST

\begin{tabular}{|c|c|c|}
\hline$U$-Number & \multicolumn{2}{|c|}{$\begin{array}{l}\text { Mg/rat for } 8 \text { days } \\
\text { Active dose Inactive dose }\end{array}$} \\
\hline 5897 & $3 \cdot 0$ & $1 \cdot 0$ \\
\hline 15,646 & $15 \cdot 0$ & $10 \cdot 0$ \\
\hline 25,352 & 15.0 & - \\
\hline 25,792 & 15.0 & - \\
\hline 25,936 & 30.0 & $15 \cdot 0$ \\
\hline 26,126 & 30.0 & 15.0 \\
\hline 26,554 & $30 \cdot 0$ & $15 \cdot 0$ \\
\hline 26,715 & $60 \cdot 0$ & $30 \cdot 0$ \\
\hline 27,045 & $15 \cdot 0$ & $5 \cdot 0$ \\
\hline 27,151 & 15.0 & - \\
\hline 27,421 & 15.0 & - \\
\hline 27,574 & 5.0 & $2 \cdot 0$ \\
\hline 27,824 & $100 \cdot 0$ & 60.0 \\
\hline 27,874 & 40.0 & 30.0 \\
\hline 27,963 & 125.0 & $100 \cdot 0$ \\
\hline 27,967 & $15 \cdot 0$ & \\
\hline
\end{tabular}

\section{REFERENCES}

Ericsson, R. J. (1970) Male antifertility compounds: U-5897 as a rat chemosterilant. F. Reprod. Fert. (In press).

ERICsson, R. J. \& BAKER, V. F. (1966) Transport of oestrogens in semen to the female rat during mating and its effect on fertility. F. Reprod. Fert. 12, 381.

ERIcsson, R. J. \& BAKER, V. F. (1970) Male antifertility compounds: biological properties of U-5897 and U-15,646. 7. Reprod. Fert. 21, 267.

Kirton, K. T., Ericsson, R. J., RAy, J. A. \& Forbes, A. D. (1970) Male antifertility compounds: efficacy of U-5897 in primates (Macaca mulatta). F. Reprod. Fert. 21, 275.

Uberoi, N. K. \& MEYer, R. K. (1966) Standardization of pregnant mare's serum. F. Reprod. Fert. 12, 587. 